\title{
«Faites votre choix en impliquant votre équipe de collaboratrices»
}

\author{
La période de recherche de places d'apprentissage a commencé: le moment oppor- \\ tun pour les médecins formateurs de se préparer aux entretiens avec les futures \\ candidates. Trois maîtres d'apprentissage totalisant ensemble pas moins de 49 ans \\ de formation donnent des conseils avertis.
}

Qu'est-ce qui vous motive à former des assistantes médicales?

Renato Tognina: Les jeunes en formation apportent un vent nouveau au cabinet et représentent un enrichissement pédagogique pour les autres assistantes diplômées. Former des assistantes médicales, c'est investir dans l'avenir. Et d'un point de vue pragmatique: lorsqu'une assistante est malade, une personne en formation peut assurer le fonctionnement du cabinet. Daniel Wildhaber: Pour moi, le contact avec les jeunes et le sentiment d'être responsable de leur formation sont source de motivation.

Verena Gantner: Je suis convaincue du système dual de formation professionnelle en Suisse et de sa qualité. Nous devons encourager la relève et dans un même temps, nous avons un devoir social envers la société de proposer des postes d'apprentissage et de permettre à de jeunes adolescentes motivées de suivre une formation professionnelle judicieuse.

Comment sélectionnez-vous vos candidates?

R. Tognina: A la lecture d'un dossier de candidature, j'essaie de voir la personne. Toutefois, je ne choisis pas de candidate dans mon rayon d'activité direct car la ville proche propose suffisamment de places de formation. Puis j'invite les candidates sélectionnées à se plonger pendant un ou quelques jours dans l'environnement professionnel du cabinet pour qu'elles rencontrent les assistantes médicales déjà en poste. C'est indispensable parce qu'il est décisif que le courant passe!

V. Gantner: Tout d'abord, j'évalue les candidatures avec mon équipe, puis nous décidons, au vu des dossiers, quelles candidates nous souhaitons inviter à un entretien. Après les entretiens, nous déterminons celles à qui nous proposons de venir faire un essai et s'immerger dans notre cabinet - c'est indispensable au moins pendant trois jours. Lorsqu'il faut départager des candidates de même niveau, ce sont les assistantes médicales qui décident. Je me réserve quand même un droit de véto.

D. Wildhaber: J'accorde une grande importance aux bulletins scolaires, la profession d'assistante médicale étant souvent sous-estimée. Beaucoup pensent que

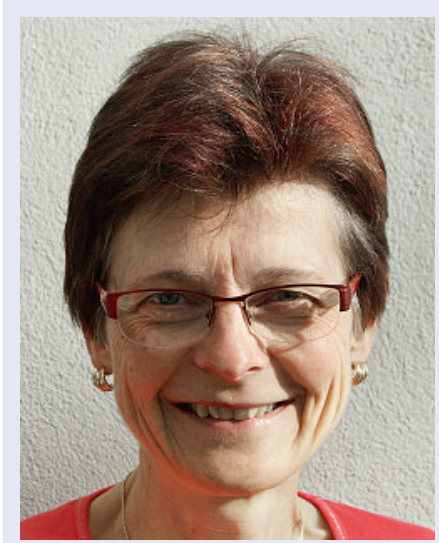

Dans son cabinet de médecine générale à Muri (AG), le Dr Verena Gantner forme des stagiaires depuis 1991 et des assistantes médicales depuis 1996. Entre 1997 et 2006, elle a dirigé la Commission chargée des examens de fin d'apprentissage.

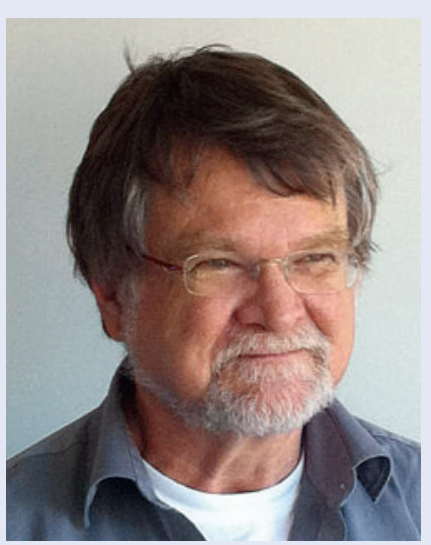

Dr Daniel Wildhaber, généraliste de Monthey, médecin formateur depuis neuf ans, a été membre de la Commis sion de réforme concernant l'Ordonnance de formation des assistantes médicales et il est actif au sein de la Commission suisse pour le développement professionnel et la qualité.

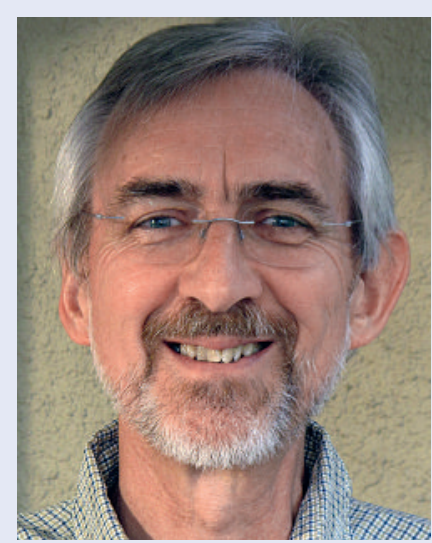

Le Dr Renato Tognina, généraliste à Steffisburg, emploie des assistantes médicales en formation depuis 1991. Il est délégué cantonal et vice-président des délégués cantonaux aux questions des assistantes médicales. 
les tâches se réduisent à passer des coups de téléphone et à taper à la machine, mais quand il s'agit de physique ou de chimie de laboratoire, il n'y a plus personne. L'entretien personnel est primordial et j'y implique mes employées. C'est en équipe que nous procédons à l'évaluation et prenons une décision.

Comment une candidate peut-elle marquer des points lors d'un entretien?

D. Wildhaber: Avec une attitude positive, et un bon mélange d'énergie et de tact, ainsi que de bonnes notes en mathématiques et en langues. Les activités extrascolaires comme des séjours linguistiques et du social sont aussi des points positifs.

V. Gantner: Pour moi ce qui compte beaucoup c'est la motivation. Il faut aussi être en mesure de la traduire en «actes», par exemple avec des connaissances préalables sur la profession ou des réactions pertinentes lors des jours d'essai. Notre préférence va aux candidates qui ont accompli une $10^{\mathrm{e}}$ année d'école ou une année intermédiaire. Les activités extrascolaires influencent aussi notre choix. Savoir exprimer ses points forts et ses points faibles lors de l'entretien laisse également une bonne impression.

R. Tognina: Pour marquer des points chez moi, les candidates doivent être spontanées, naturelles. L'organisation du temps libre, et des loisirs sportifs, créatifs ou sociaux, en dit aussi long sur une personne, sur son engagement, sa persévérance ou son envie de performance.

Quels conseils donner à des collègues qui engagent pour la première fois une assistante en formation?

V. Gantner: Procédez à une évaluation approfondie des candidates en collaboration avec l'assistante médicale responsable. N'engagez jamais quelqu'un pour faire plaisir à un ami ou à un collègue, mais uniquement lorsque vous êtes vous-même convaincu.

D. Wildhaber: Prenez votre décision en concertation avec votre équipe. N'engagez pas une jeune en formation pour économiser une assistante médicale. Lorsque vous choisissez une candidate parmi vos proches ou les proches de vos patients, pensez au secret médical mais aussi à ce qu'elle puisse suivre sa formation sans être prise dans un étau. Gardez un juste équilibre entre critiques et compliments.

R. Tognina: Motivation, ouverture et persévérance justement aussi pendant les activités du temps libre comptent plus qu'un bulletin scolaire exceptionnel.

Selon vous, qu'est-ce qui est essentiel pour la formation des assistantes médicales à l'avenir?

R. Tognina: Les médecins doivent savoir que la formation de bonnes collaboratrices pour leurs cabinets dépend aussi d'eux.

D. Wildhaber: La formation des assistantes médicales doit être interdisciplinaire. Au quotidien, celles-ci associent en effet leur savoir-faire acquis dans diffé- rents domaines: un ECG par exemple demande qu'elles accueillent aimablement le patient, le renseignent de manière compétente et éventuellement le calment tout en prenant en compte les aspects pathologiques, laboratoires, thérapeutiques et pharmacologiques de l'examen médical. Pour augmenter l'attrait de cette profession, il serait aussi opportun de réfléchir aux salaires qui sont trop modestes.

V. Gantner: Si c'était possible, tous les cabinets devraient former des assistantes. Avec de la bonne volonté, pratiquement tous les cabinets peuvent créer un poste de formation. Les échanges permettent aux assistantes de pratiquer des activités qui ne sont pas exercées dans le cabinet d'apprentissage. Le contact avec des jeunes motivées est plaisant et permet au formateur de rester jeune!

\section{Aide-mémoire «Sélection du personnel dans un cabinet médical»}

Le choix de la collaboratrice ou de l'assistante en formation idéale peut soulever des questions chez les médecins. C'est pour cette raison que la fondation Margrit et Ruth Stellmacher, une institution pour le soutien et l'encouragement de la profession d'assistante médicale, a publié cet aide-mémoire en 2003. En collaboration avec I'Institut de psychologie de I'Université de Berne, elle a ainsi créé un outil permettant d'évaluer les principales activités et compétences d'une assistante médicale.

La généraliste Verena Gantner a contribué à la réalisation de cet aide-mémoire. Elle précise sa motivation: «Un processus de sélection amélioré permet d'éviter les interruptions en milieu d'apprentissage et la frustration qui y est liée pour l'assistante en formation et le formateur.» Cet aide-mémoire a été créé en premier lieu pour aider à choisir les personnes qui souhaitent suivre une formation d'assistante médicale mais il est aussi utile pour engager une collaboratrice diplômée. «Son avantage est de proposer une marche à suivre structurée pour procéder à la sélection des candidates» explique Verena Gantner. Par ailleurs, il aide à garder présent à l'esprit l'ensemble des compétences requises et à les tester lors d'un entretien ou pendant quelques jours d'essai.

Et même si ce document est trop détaillé pour beaucoup de cabinets, Verena Gantner reste convaincue: «C'est ainsi plus facile de définir les compétences spécifiques à un cabinet et, pour le formateur, d'évaluer une candidate avec précision.» L'aide-mémoire a en outre fait ses preuves lors de la demande de bourse pour écoles privées. Des cabinets testent les demandeurs de bourse sur la base de l'aide-mémoire et rédigent ensuite des recommandations.

L'aide-mémoire «Sélection du personnel dans un cabinet médical» est disponible gratuitement sur le site de la FMH: www.fmh.ch $\rightarrow$ Service $\rightarrow$ Assistantes médicales $\rightarrow$ Documents à imprimer 